\title{
Semianalytical Universal Simulation of the Electrical Properties of the Permeable Base Transistor
}

\author{
P. Chenevier, G. Kamarinos, and G. Pananakakis \\ LPCS - URA CNRS 840 - Enserg \\ 23 rue des Martyrs, BP 257, F-38016 Grenoble Cédex, FRANCE
}

\begin{abstract}
Using only a few numerical calculations, we give the analytical currentvoltage and charge-voltage characteristics valid for any PBT. The highest unity current gain frequency $\left(\mathrm{f}_{\mathrm{T}}\right)$ corresponding to the current technology is on the order of $30 \mathrm{GHz}$; nevertheless, the oscillation frequency can be higher than $100 \mathrm{GHz}$.
\end{abstract}

\section{Introduction}

There are two types of PBT : the buried base PBT, and the etched groove PBT [1]

The PBT is essentially a two dimensional structure ; it is so impossible to find $a b$ initio analytical expressions for its characteristics. The optimization of the device needs time consuming 2-D programs, and in particular, concerning its frequency limits.

In this paper, we show, for the first time, that the modelization of the PBT do not require to have a continuous recourse to a 2-D numerical simulation program, and we give a definitive answer to the high frequency performances of the device. To reach these results : (i) we have worked in the buried base PBT, and (ii) we have utilized the 2-D numerical program (TITAN+JUPIN) of CNET/CNS. Evidently, the PBT is a short channel MESFET. So, we have studied the half period of the structure $\left(L=\frac{a+d}{2}\right.$; Fig. 1), and we have supposed that the device is limited in its active zone : $\mathrm{W}_{\mathrm{E}}+\mathrm{W}_{\mathrm{C}}$; the device bias are then applied on the limits of these zones $\left(\mathrm{V}_{\mathrm{BE}}^{\prime}\right.$ and $\left.\mathrm{V}_{\mathrm{CE}}^{\prime}\right)$.

\section{Similitude Laws for Low Collector Bias}

We suppose here that in the electron velocity expression : $\vec{V}_{n}=\mu_{n} \vec{E}$, the mobility is constant. Besides, we adopt the classical hypothesis : (i) SCR totally depleted, (ii) at SCR boundaries, $\frac{\partial \phi}{\partial \vec{n}}=0$, and (iii) into the channel $n \approx N_{D}$.

The study of Poisson equation with its boundary conditions shows that, if one takes $\frac{d}{2}$ as the length unity, and $\frac{q N_{D}}{\varepsilon} \frac{d^{2}}{4}$ as the potential unity, the potential $\phi(X, Y)$ depends only 
on the two dimensionless parameters $X=\frac{2 W_{E}}{d} ; Y=\frac{2 W_{C}}{d}$, where $\mathrm{W}_{\mathrm{E}}$ and $\mathrm{W}_{\mathrm{C}}$ are the lengths of SCR around the base :

$$
W_{E}=\left[\frac{2 \varepsilon}{q N_{D}}\left(V_{D}-V_{B E}^{\prime}\right)\right]^{1 / 2} ; W_{C}=\left[\frac{2 \varepsilon}{q N_{D}}\left(V_{D}-V_{B E}^{\prime}+V_{C E}^{\prime}\right)\right]^{1 / 2}
$$

We show then that the transistor current can be written as :

$$
I_{C}=-\frac{q^{2} N_{D}^{2} \mu_{n} Z d^{2}}{4 \varepsilon} f\left(\frac{2 W_{E}}{d}, \frac{2 W_{C}}{d}\right)
$$

To determine the function $f(X, Y)$, it is sufficient to plot the $I_{C}\left(V_{B E}^{\prime}, V_{C E}^{\prime}\right)$ characteristics for only one set of technological parameters $\left(\frac{d}{2}, N_{D}, \mu_{n}\right)$. Therefore, using the 2-D numerical program for calculating the current characteristics of only one (non particular) PBT, we can calculate analytically the characteristics of any other device.

The threshold voltage of PBT (for $\mathrm{V}_{\mathrm{CE}}=0$ ) can also be calculated :

$$
l(0)=H(0) W_{E} ; V_{B E T}=V_{T} \text { for } \frac{d}{2}-l(0)=0, \text { so, } V_{T}=V_{D}-\frac{q}{2 \varepsilon H^{2}} N_{D} d^{2}
$$

The simulated characteristics give : $\mathrm{H}(0) \approx 0,7$.

\section{High Collector Bias Regime}

The used model takes $\vec{V}_{n}=V_{s} \frac{\vec{E}}{E+E_{C}}$ with $V_{s}=1.04 \times 10^{7} \mathrm{~cm} \cdot \mathrm{s}^{-1} ; E_{C}=1.04 \times 10^{4} \mathrm{Vcm}^{-1}$ The current $I_{C}$ can be written :

$$
I_{C}=-q N_{D} Z V_{m}\left[\left(\frac{d}{2}-h\right)\right]
$$

where $\mathrm{V}_{\mathrm{m}}$ is an average velocity; $\mathrm{V}_{\mathrm{m}}$ and $\mathrm{h}$ are, a priori, dependent from bias, doping $\left(\mathrm{N}_{\mathrm{D}}\right)$ and $\frac{d}{2}$. Using the 2-D program, we show that : (i) $\mathrm{V}_{\mathrm{m}}$ and $\mathrm{h}$ are independent from $\frac{d}{2}$, (ii) $\mathrm{V}_{\mathrm{m}}$ is independent from $\mathrm{V}_{\mathrm{BE}}^{\prime}$, (iii) we can write $: h=H\left(N_{D}, V_{C E}^{\prime}\right) W_{E}$,

(iv) $H=0.705-0.0525 V_{C E}^{\prime}$

so $\mathrm{H}$ is, in a first approximation, independent from $\mathrm{N}_{\mathrm{D}}$,

(v) satisfactory analytical expression for $\mathrm{V}_{\mathrm{m}}$ is :

$$
V_{m}=\frac{V_{C E}^{\prime}}{V_{C E}^{\prime}+E_{C}\left(W_{E_{o}}+W_{C_{o}}\right)}
$$

$\mathrm{W}_{\mathrm{E}_{0}}$ and $\mathrm{W}_{\mathrm{C}_{0}}$ being the values for $\mathrm{V}_{\mathrm{BE}}=0$.

We establish so a universal expression for $\mathrm{I}_{\mathrm{C}}$ :

with $\mathrm{V}_{\mathrm{m}}$ and $\mathrm{H}$ given by (6) and (5).

$$
I_{C}=-q N_{D} Z V_{m}\left[\frac{d}{2}-H W_{E}\right]
$$

These analytical expressions fit very satisfactorily the 2-D simulations (Fig. 2 for example, where the dots correspond to the analytically calculated current). The 2-D simulations allow us to find also an analytical expression for the total charge in the SCR : 


$$
Q_{S C}=q N_{D} Z S=q N_{D} Z\left(W_{E}+W_{C}\right)\left[\frac{a}{2}+\alpha_{0} \frac{W_{E}\left(W_{E}+W_{E}\right)}{3 W_{E}+W_{C}}\right]
$$

we find $\alpha_{0}=0.77$.

\section{Small Signal Parameters; Frequency Limits}

Using the analytical expressions of current (7) and charge (8), we can calculate : the conductance $g_{D}$, the transconductance $g_{m}$ and the interelectrode capacitances $C_{B E}$ and $\mathrm{C}_{\mathrm{BC}}$. The unity current gain (transition) frequency is then calculated :

$$
\begin{gathered}
f_{T}=\frac{g_{m}}{2 \pi\left(C_{B E}\left(C_{B E}+2 C_{B C}\right)\right)^{1 / 2}} \\
g_{m}=Z \frac{\varepsilon}{W_{E}} V_{m} H \\
\left.C_{B E}=Z \frac{\varepsilon}{W_{E}}\left[\left(\frac{a}{2}\right)+\alpha_{o} W_{C} \frac{(1+\gamma)}{(1+3 \gamma)^{2}}\left(6 \gamma^{2}+3 \gamma+1\right)\right] \quad\right) \\
C_{B C}=Z \frac{\varepsilon}{W_{E}}\left[\left(\frac{a}{2}\right) \gamma+\alpha_{0} W_{C} \frac{(1+\gamma)}{(1+3 \gamma)^{2}} \gamma^{2}(5 \gamma+1)\right] \\
\left.\gamma=\frac{W_{E}}{W_{C}}=\left[\frac{V_{D}-V_{B E}^{\prime}}{V_{D}-V_{B E}^{\prime}+V_{C E}^{\prime}}\right]^{1 / 2}\right)
\end{gathered}
$$

\section{Conclusion and Remarks}

The $f_{T}$ corresponding to the Fig. 2 parameters, is not higher than $30 \mathrm{GHz}$ (Fig. 3). This result is confirmed by all the published experimental works $[2,3,4,5,6,7]$; the PBT on $\mathrm{GaAs}$ has the same limits $\left(\mathrm{V}_{\mathrm{s}}\right.$ is nearly the same for $\mathrm{Si}$ and $\left.\mathrm{GaAs}\right)$; the etched groove PBT can be slightly better $\left(\mathrm{C}_{\mathrm{BE}}\right.$ is lower) ; the maximum frequency oscillations, according to our estimations, must be higher than $100 \mathrm{GHz}$; so, the PBT, as it can deliver an important power, can be an interesting device for high frequency power amplification [8].

\section{References}

[1] B.J. VOJAC, G.D. ALLEY - IEEE Trans. ED-30, 877 (1983)

[2] B.O. BOZLER - Surf. Science, 174, 487 (1986)

[3] K.B. NICHOLS et al - IEEE Trans. ED-35, 2246 (1988)

[4] D.D. RATHMAN, W.K. NIBLACK - MTT Int. Microw. Symp., Dig.1, 357 (1988)

[5] L.J. KUSHER - Microw. J., 33, 87 (1990)

[6] T. OSHIMA et al - IEDM Proceed. (IEEE ed.) 33 (1991)

[7] A. GRUHLE et al - ESSDERC'91 Proceed., Elsevier ed., 27 (1991)

[8] P. CHENEVIER, G. PANANAKAKIS, G. KAMARINOS, F. STEINHAGEN Superl. \& Microst., 8, 269 (1990) 

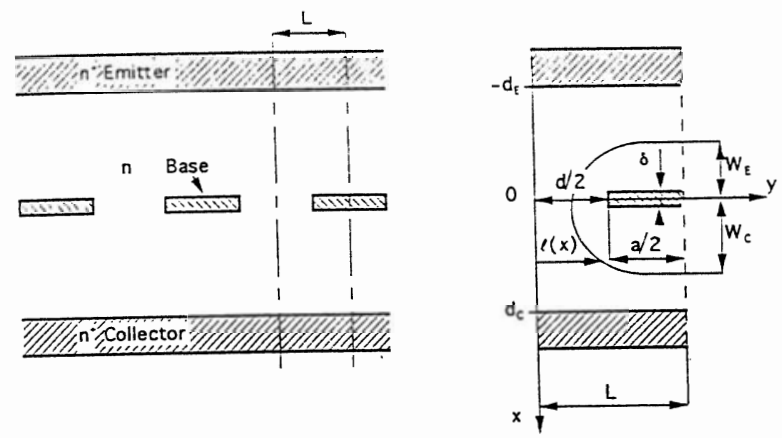

Fig. 1

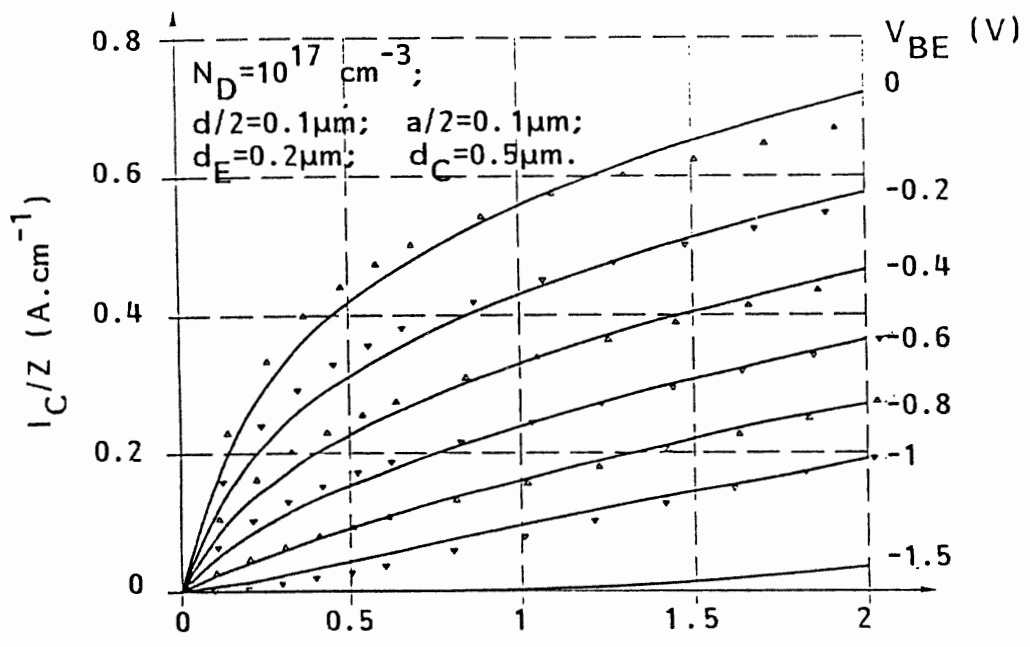

Fig. 2

$$
V_{C E}(V)
$$

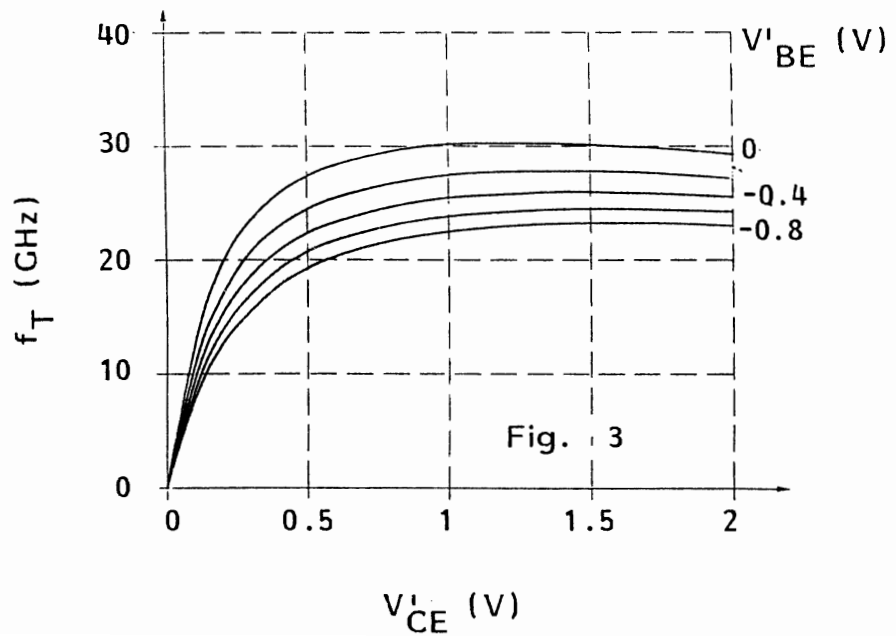

